\title{
Numerical Simulations for a Partial Disk MHD Generator Performance
}

\author{
Lai Li ${ }^{1}$, Hu-lin Huang ${ }^{2, *}$ (i) and Gui-ping Zhu ${ }^{2}$ \\ 1 College of Power and Energy Engineering, Nanjing University of Aeronautics \& Astronautics, \\ Nanjing 210016, China; 112015js@nuaa.edu.cn \\ 2 College of Astronautics, Nanjing University of Aeronautics \& Astronautics, Nanjing 210016, China; \\ zhuguiping@nuaa.edu.cn \\ * Correspondence: hlhuang@nuaa.edu.cn; Tel.: +86-025-8489-6183
}

Received: 15 November 2017; Accepted: 31 December 2017; Published: 4 January 2018

\begin{abstract}
In this paper, numerical simulations are carried out to predict the performance of a new designed configuration of the disk magnetohydrodynamic (MHD) generator, which segments the generator into dozens of parts. The behaviors and characteristics of segments are mainly investigated with number of parts at 24, 36, 60, 72, 90 adopted Large Eddy Simulation (LES). The numerical results declared that these division generators approach more stable plasma ionization and better performance than that of the conventional disk MHD generator at the same working conditions. The optimal value can be reached when the angle is 5-10 degrees (36-72 parts). Due to the division of the generator, the internal resistance is larger than that of the conventional disk channel that causes the reduction of Faraday current, hence the Lorentz force, $j_{\theta} B$, decreased. Therefore, the radial velocity increased and static pressure decreased. Consequently, the reduction of static pressure contributes to improvement to the plasma uniformity and ionization stability. Those features reveal that the designed configuration has the obvious advantage on raising energy conversion efficiency and power output.
\end{abstract}

Keywords: disk magnetohydrodynamic (MHD) generator; high temperature plasma; inert gas; energy conversion

\section{Introduction}

Disk magnetohydrodynamic (MHD) power generator has been widely investigated in the past few decades due to its high-efficiency on converting the thermal energy of a working gas into electrical power. However, at the normal working temperature, it is hard to maintain an adequate electrical conductivity to form current loop. So, one familiar to enhance the electrical conductivity is adding some alkali seeds into the working gas [1-3]. The disadvantage of this method is the system need to add extra devices for the seeds recycling. The seeds often gathered together on the wall, which would deteriorate generator performance and even cause the channel eroded. The other way to enhance the electrical conductivity was to elevate the gas temperature. Inert mixture gas $(\mathrm{He} / \mathrm{Xe})$ plasma without any alkali seeds has been proposed and demonstrated as the working gas under a high temperature condition in recent years. Harada [4,5] investigated plasma stability of the inert gas in the disk MHD generator and found that the non-uniform discharge structure caused deterioration to the generator performance, increased local current concentration, enhanced local Lorentz force, and decreased the gas velocity, increased the static gas pressure. According to Harada's results, the decrease of static gas pressure is caused by the smaller Lorentz force resulting from the reduction of the tangent current. He pointed out that the smaller static pressure was beneficial for ionization stability and generation performance [6]. Namely, the weaker tangential current is helpful to reduce the Lorentz force and static pressure. Okuno and Murakami investigated the characteristics and performance of the disk 
generator by numerical simulations and experiments using seed-free high temperature argon gas $[7,8]$. They studied the influence of total inflow temperature between 7600-9600 K for the argon plasma. They concluded that the higher total inflow temperature could significantly improve plasma stability state and the power generation performance, even under low-density magnetic flux. The obtained generator performance was comparable or superior to previous results that were obtained by using a conventional low-temperature gas with seeds. Tanaka, M. [9] studied the effects of the self-excited Joule heating in the nozzle region of a seed-free inert-gas disk-shaped magnetohydrodynamic (MHD) generator. He concluded that the high current density around the anode edge can induce a vertical inhomogeneity in the ionization degree that causes a boundary layer to develop. Owing to the higher ionization rates, the inhomogeneity of the plasma and the increase in the ionization degree in the nozzle are more marked for argon and xenon, than for helium. He also pointed out that the electron temperature and the power output become stable with the increase in the total inlet temperature to $9000 \mathrm{~K}$ and a recombination process in the plasma is dominant, which can provide homogeneous and stable plasma and suppress the streaky and unstable behavior observed at a low inlet temperature of $7500 \mathrm{~K}$ [10]. Based on these conclusions, the high total inlet temperature is chosen in our researches.

On the other side, the channel structure also affects the performance of the generator. Liberati, A proposed a contoured disk walls profile. He found that was useful to improve the performance of the generator [11]. Then, a less-divergent (small area ratio) [12] and convexly divergent [13] walls profile was studied. To reduce the static pressure and the effects of the Lorentz force, the positive inlet swirl was introduced [14]. These works were theoretically valid to improve the generator performance with both seeded and seed free flow.

Gas flows can be categorized into different flow regimes based on the Knudsen number Kn. Therefore, it is important to adopt the adequate model. In the continuum regime $(\mathrm{Kn}<0.001)$, the Navier-Stokes equations (NSE) are adequate to describe the fluid behavior. In the near continuum regime $(0.001<\mathrm{Kn}<1)$, the NSE are known to lose accuracy or be inadequate [15]. In the transition flow regime $(0.1<\mathrm{Kn}<10)$, the NSE with slip boundary conditions are adopted. Liu, Hongwei, et al. presented a gas-kinetic finite volume scheme for the numerical solution of the multiple temperature kinetic model (MTKM) in the continuum and transition flow regimes [15]. When the gas density is not very high, the direct simulation Monte Carlo (DSMC) method is one of the most successful techniques for the gas flows. T. Zhu and W. Ye [16] studied the non-continuum gas-phase heat conduction in micro/nano devices by the DSMC method. The lattice-Boltzmann (LB) method is widely adopted as a numerical tool for computational fluid dynamics, due to its simple formulation and application to flow problems when compared with solving the NSE [17]. In this paper, the Knudsen number is quite smaller than 0.001, the NSE model is adopted.

In this paper, a new designed channel structure was proposed to weaken the tangential current. The disk generator was segmented into $24,36,60,72,90$ parts with its corresponding division angle $15,10,6,5,4$ degrees, respectively. To investigate the performance and plasma characteristics of the new designed generator, the numerical simulations were carried out for high temperature argon gas without any alkali seeds. The structure influence on the generator performance was discussed. The results showed that the optimal angle of the new designed channel could achieve more stable plasma ionization and approach better power generation performance than the general disk MHD generator does.

\section{Numerical Model}

\subsection{Numerical Simulation Domain}

Figure 1a illustrates the structure of the new designed generator channel. The generator is divided into dozens of independent parts. The structure of each part is in same, and for convenience, is named as the partial disk generator. The inflow from the entrance as shown in Figure 1a is accelerated by the convergent-divergent configuration of the channel throat region. After flowing through the MHD 
channel, the working gas is recycled or discharged from the system. Figure $1 \mathrm{~b}$ shows the scale of channel cross section in xoz plane ( $r-z$ in cylinder coordinate).

Figure 1. Structure of generator: (a) Schematic of a divided disk magnetohydrodynamic (MHD) generator; and, (b) Channel cross-section.

\subsection{Mathematical Model}

In the research model, the working gas is assumed to be pure noble gas. The governing equations of state equations and Maxwell equations are in combination to describe the non-equilibrium plasma. The non-equilibrium plasma in the generator channel consists of ions, electrons, and atoms under two-temperature model [18]. The Navier-Stokes equations and the energy equation include Lorentz force and Joule heating terms, respectively. Large Eddy simulation (LES) is used to describe the flow.

\subsubsection{Governing Equations for MHD Plasma Flows.}

The time dependent compressible Navier-Stokes equations, coupled with Lorentz force and Joule heating terms, are the following [8]:

Continuity equation:

$$
\frac{\partial \rho}{\partial t}+\nabla \cdot(\rho \mathbf{u})=0
$$

Momentum equation:

$$
\frac{\partial \rho \mathbf{u}}{\partial t}+\nabla \cdot(\rho \mathbf{u u})=\mathbf{j} \times \mathbf{B}-\nabla p+\nabla \cdot \boldsymbol{\tau}
$$

Energy equation:

$$
\begin{aligned}
\frac{\partial E_{s}}{\partial t}+\nabla \cdot\left(E_{s}+p\right) \mathbf{u} & =\mathbf{u} \cdot(\mathbf{j} \times \mathbf{B})-\nabla \cdot \mathbf{q}+\frac{|\mathbf{j}|^{2}}{\sigma}+\nabla \cdot(\boldsymbol{\tau} \mathbf{u}) \\
E_{s} & =\rho\left(c_{V} T+\frac{1}{2} \mathbf{u}^{2}\right)
\end{aligned}
$$

where $\rho$ is mass density, $\mathbf{B}$ is the magnetic flux density vector, $\mathbf{u}$ is the velocity vector of flow, $c_{V}$ is the constant volume specific heat, $T$ is the static temperature, $\mathbf{j}$ is the current density vector, $E_{S}$ is the total energy, $p$ is the static pressure, $\sigma$ is the electrical conductivity, $\mathbf{q}$ is the conductive heat flux vector, and $\tau$ is the viscous stress.

2.2.2. Governing Equations for Charged Particles.

Conservation of ion number density:

$$
\begin{gathered}
\frac{\partial n_{i}^{+}}{\partial t}+\nabla \cdot n_{i}^{+} \mathbf{u}=\dot{n}_{i}^{+}=k_{f i} n_{e} n_{i}-k_{r i} n_{e}^{2} n_{i}^{+} \\
n_{e}=\sum_{i}^{i o n} n_{i}^{+}
\end{gathered}
$$

Energy equation for electron:

$$
\begin{gathered}
\frac{\partial U_{e}}{\partial t}+\nabla \cdot\left(U_{e} \mathbf{u}_{e}\right)=\frac{|\mathbf{j}|^{2}}{\sigma}-p_{e} \nabla \cdot \mathbf{u}_{e}-3 n_{e} k\left(T_{e}-T\right) \sum_{h}^{\text {heavy }} \frac{m_{e}}{m_{h}} \bar{v}_{e h} \\
p_{e}=n_{e} k T_{e}, U_{e}=\frac{3}{2} n_{e} k T_{e}+n_{i}^{+} \varepsilon_{i}
\end{gathered}
$$


In Equation (5), $n_{i}^{+}$is the number density of argon ions, $n_{e}$ is the number density of electron, $n_{i}$ is the number density of argon atom, and the three-body recombination rate coefficient $k_{r}$ is determined by the following relations [10]:

$$
\begin{gathered}
k_{r}=k_{r h} k_{r o} /\left(k_{r h}+k_{r o}\right) \\
k_{r h}=1.09 \times 10^{-20} T_{e}^{-9 / 2} \\
k_{r o}=3.33 \times 10^{-44}\left(\frac{135300}{T_{e}}+2\right) \exp \left(\frac{47800}{T_{e}}\right)
\end{gathered}
$$

the ionization rate coefficient $k_{f}$ is derived on the basis of the principle of detailed balance with the Saha equilibrium:

$$
k_{f} n_{i} n_{e}=k_{r} n_{i} n_{e}^{2}, k_{f}=k_{r} \times \frac{g_{i}}{g_{0}}\left(\frac{2 \pi m_{e} k T_{e}}{h^{2}}\right)^{3 / 2} \exp \left(-\frac{\varepsilon_{i}}{k T_{e}}\right)
$$

where $g_{i}$ is the statistical weight of the ground state of the ion, $g_{0}$ is the statistical weight of the ground state of the neutral argon atom, $\varepsilon_{i}$ is ionization potential of argon atom $[10,18]$, the subscripts $e, i$ and the superscript + denote the electrons, neutral atoms, and ionized particles, respectively. In Equation (7), $U_{e}$ is the electron energy, $\mathbf{u}_{e}$ is the velocity vector of electrons, $m_{h}$ is the mass of heavy particle, $m_{e}$ is the mass of electron, and $p_{e}$ is the electron pressure. In Equation (8), $k$ is Boltzmann constant, $T_{e}$ is electron temperature, and $\varepsilon_{i}$ is the ionization energy.

\subsubsection{Governing Equations for Electrical-Magnetic}

Generalized Ohm's law:

$$
\begin{gathered}
\mathbf{j}+\frac{\beta}{|\mathbf{B}|} \mathbf{j} \times \mathbf{B}=\sigma(\mathbf{E}+\mathbf{u} \times \mathbf{B}) \\
\beta=\frac{e|\mathbf{B}|}{m_{e} \bar{v}_{e h}}
\end{gathered}
$$

Maxwell equations:

$$
\begin{gathered}
\nabla \times \mathbf{H}=\mathbf{j}+\frac{\partial \mathbf{D}}{\partial t}, \nabla \cdot \mathbf{B}=0 \\
\nabla \times \mathbf{E}=-\frac{\partial \mathbf{B}}{\partial t}, \nabla \cdot \mathbf{D}=\rho_{e}
\end{gathered}
$$

Since the charge neutrality is assumed and magnetic Reynolds number is small enough (about $0.082 \ll 1)$, the Maxwell equations are simplified as follows:

$$
\nabla \times \mathbf{E}=0, \nabla \cdot \mathbf{j}=0
$$

Equation (9) is the general Ohm's law, In Equation (10), $\bar{v}_{e h}=\sum_{h}^{h e a v y} n_{h} Q_{e h} c_{e}$ is the average momentum transfer collision frequency for an electron e with a heavy particle $h$, where $Q_{e h}$ is the energy-averaged momentum transfer cross section, $C_{e}=\sqrt{8 k T_{e} / \pi m_{e}}$ is the mean electron velocity for a Maxwellian distribution, $\beta$ is Hall parameter. In Equation (12), $\mathbf{E}=-\nabla \Phi$ is the electric field vector, where $\Phi$ is the electrical potential.

\subsection{Boundary Conditions and Numerical Procedures}

The codes and numerical procedures of simulation are firstly verified by comparing the results of literature (Ref. 7) (Figure 2). The comparison shows that, within the acceptable range of error, the code and the program are effective. 


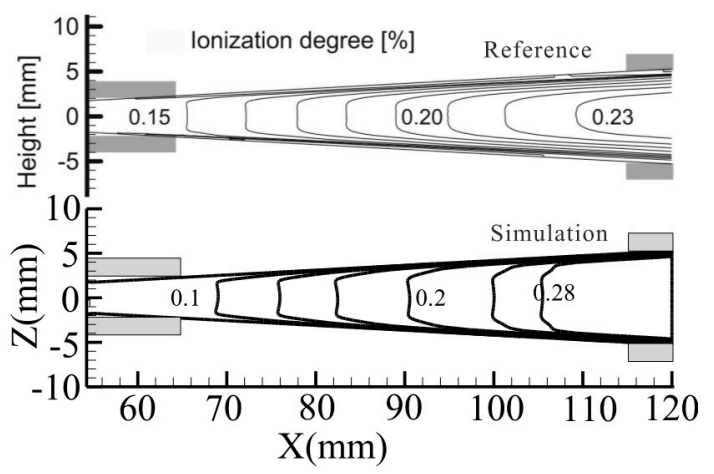

(a)

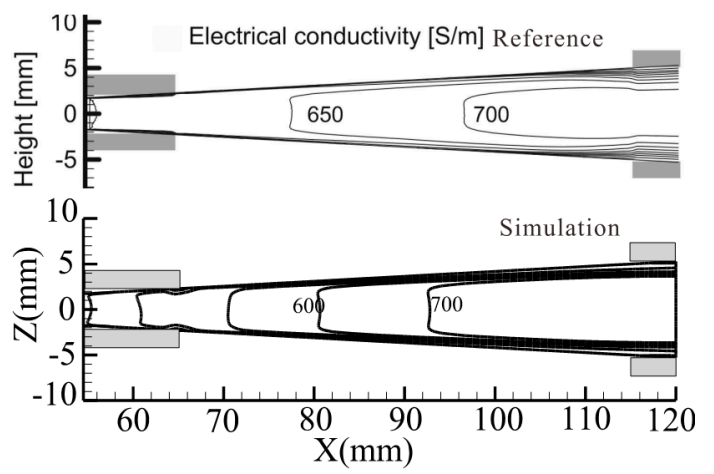

(b)

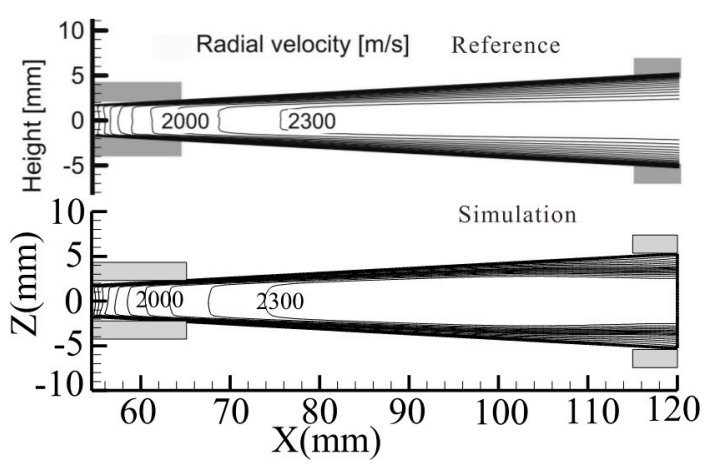

(c)

Figure 2. The verification of the simulations: (a) Ionization degree; (b) Electrical conductivity; (c) Radial velocity.

The non-equilibrium seed-free plasma as working gas in Faraday-Type and Disk-Type generator channel have been widely studied [19-28]. In this paper, high-temperature seed-free argon plasma is used. Table 1 shows the working conditions in the simulation processes. A non-slip wall condition is applied as the boundary condition for the flow. The wall of the partial channel is electrically insulating, and its temperature is set to be $300 \mathrm{~K}$. The boundary condition for the electrical equation is given as $\Phi=0$ on the anode and $\Phi=\mathrm{V}_{\mathrm{h}}$ on the cathode, where $\mathrm{V}_{\mathrm{h}}$ is the Hall voltage. As shown in Figure 1b, the throat height and the cathode inlet height are $3.55 \mathrm{~mm}(x=54.5 \mathrm{~mm})$ and $9.87 \mathrm{~mm}$ $(x=115 \mathrm{~mm})$, respectively. With the division angle expanding at the value of $4,5,6,10$, and 15 , the corresponding arc lengths in outlet $(125 \mathrm{~mm})$ are $8.72 \mathrm{~mm}, 10.9 \mathrm{~mm}, 13.08 \mathrm{~mm}, 21.81 \mathrm{~mm}$, and 32.71 $\mathrm{mm}$, respectively. The corresponding grid numbers of the channel are $40 \times 120 \times 50,40 \times 120 \times 60$, $40 \times 120 \times 70,40 \times 120 \times 100,40 \times 120 \times 130$ in Cartesian coordinate on $z \times x \times y$ direction, respectively. The location of electrode regions varies from 54.5 to $65 \mathrm{~mm}$ for the anode, 115 to $120 \mathrm{~mm}$ for the cathode. The external load resistance for $4,5,6,10$, and 15 are $0.68,0.65,0.64,0.61$, and $0.58 \Omega$, respectively. The LES-Smagorinsky model is applied as turbulence model to solve the Equations (1)-(3) for the description of the flow. The Equations (5), (7), and (8) are solved by means of the user defined functions code for Fluent. The elliptic equations for the potential derived from (9), (13) is solved MHD model of Fluent. The set of above equations is solved numerically by means of the first-order upwind scheme. 
Table 1. Conditions used in calculation.

\begin{tabular}{cc}
\hline Working fluid & $\mathrm{Ar}$ \\
Total inflow pressure & $0.16 \mathrm{MPa}$ \\
Total inflow temperature & $9200 \mathrm{~K}$ \\
Inlet static temperature & $8000 \mathrm{~K}$ \\
Inlet electron temperature & $8000 \mathrm{~K}$ \\
Magnetic flux density & $1 \mathrm{~T}$ \\
\hline
\end{tabular}

\subsection{Grid Meshes Selection}

The numerical region is one of the 15 degrees of segmentation, its total mesh number is 624,000 $(40 \times 120 \times 130)$. It was selected from four different sets of grids mesh number, which are 260,000 $(40 \times 100 \times 65), 380,000(40 \times 100 \times 95), 520,000(40 \times 100 \times 130)$, and $624,000(40 \times 120 \times 130)$, respectively. Figure 3 shows that the selected mesh numbers reduce the coarsening effect of the grid with 260,000 mesh numbers and its results were no significant differences with that of 520,000. From Table 2, it shows that the value of variables at the location of $80 \mathrm{~mm}$ of the channel has very little differences for the mesh numbers of 520,000 and 624,000. Hence, the largest mesh numbers is adopted in the channels of the other angles.

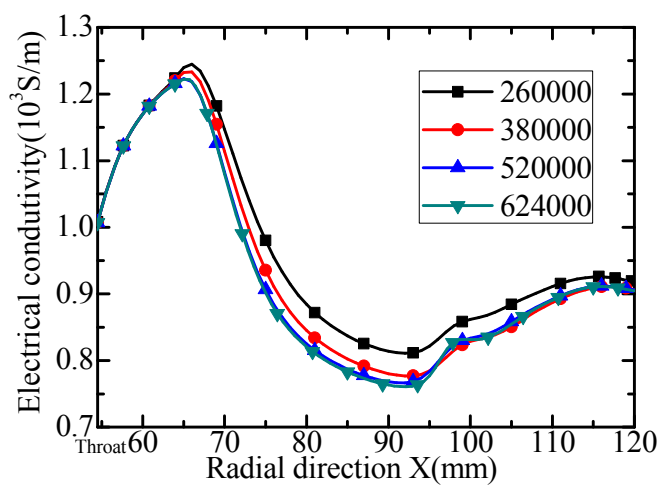

Figure 3. The comparison of electrical conductivity of the different mesh numbers.

Table 2. Value of variables in the different mesh numbers at $80 \mathrm{~mm}$ of the channel.

\begin{tabular}{ccccc}
\hline Mesh Numbers & $\mathbf{2 6 0 , 0 0 0}$ & $\mathbf{3 8 0 , 0 0 0}$ & $\mathbf{5 2 0 , 0 0 0}$ & $\mathbf{6 2 4 , 0 0 0}$ \\
\hline Electrical conductivity $\left(10^{3} \mathrm{~S} / \mathrm{m}\right)$ & 0.88 & 0.85 & 0.83 & 0.82 \\
Velocity $\left(10^{3} \mathrm{~m} / \mathrm{s}\right)$ & 2.09 & 2.11 & 2.14 & 2.144 \\
Static temperature $\left(10^{3} \mathrm{~K}\right)$ & 4.32 & 4.23 & 4.15 & 4.14 \\
Electron temperature $\left(10^{3} \mathrm{~K}\right)$ & 6.78 & 6.52 & 6.38 & 6.35 \\
\hline
\end{tabular}

\section{Simulation Results and Discussion}

\subsection{Comparison of Simulation Results between Partial Disk and General Disk Generator}

There are three kinds of current in the disk generator channel, one is in the radial direction:

$$
j_{r}=\frac{\sigma}{1+\beta^{2}}\left(E_{r}-\beta E_{\theta}+\beta u_{r} B+u_{\theta} B\right)
$$

The second is in the tangential direction:

$$
j_{\theta}=\frac{\sigma}{1+\beta^{2}}\left(\beta E_{r}+E_{\theta}-u_{r} B+\beta u_{\theta} B\right)
$$


The third is in the $z$ direction:

$$
j_{z}=\sigma E_{z}
$$

where $E_{r}, E_{\theta}$ are the component of electric field in $r, \theta$ directions, respectively; $j_{r}, j_{\theta}, j_{z}$ are the component of current density in radial, tangential, $z$ directions, respectively; $u_{r}, u_{\theta}$ are the component of flow velocity in the radial, tangential directions, respectively.

For the channel is segmented, the internal resistance is larger than that of general disk channel, which causes the decrease of Faraday current. So, the radial velocity increases due to the Lorentz force decreasing. Under the influence of the side wall in the partial channel, the tangential velocity is reduced. It can be deduced from (14) and (15) that the radial current is enhanced, meanwhile, the tangential current is weakened. Thus, the radial velocity is higher, and the static pressure is smaller in the partial channel than that in the general disk channel.

The external magnetic field is only applied along the z-direction and induced field can be neglected owing to low Reynolds' number, so the influence of Lorentz force and current along z-direction can be ignored. The Lorentz force along tangential direction is not discussed here due to it being perpendicular to the main flow direction with little influence on the radial velocity. Figure 4 shows the comparison of the tangential current between the partial disk generator $\left(\theta=6^{\circ}\right)$ and the general disk generator, where the negative sign means the reverse direction. As shown in Figure 4, the maximum magnitudes of the tangential current in the partial disk generator and the general disk generator are about $0.9 \times 10^{6} \mathrm{~A} / \mathrm{m}^{2}$, $1.2 \times 10^{6} \mathrm{~A} / \mathrm{m}^{2}$, respectively. Along the MHD region of the channel $(54.5-120 \mathrm{~mm})$, the later quantity is consistently larger than that of the former. Although the average electrical conductivity in the general disk is superior (Figure 5), the term of current is in dominant. As the formula of Joule heating $j^{2} / \sigma$ shown, the Joule heating effect is stronger in the general disk channel, which is the main energy resource of plasma ionization.

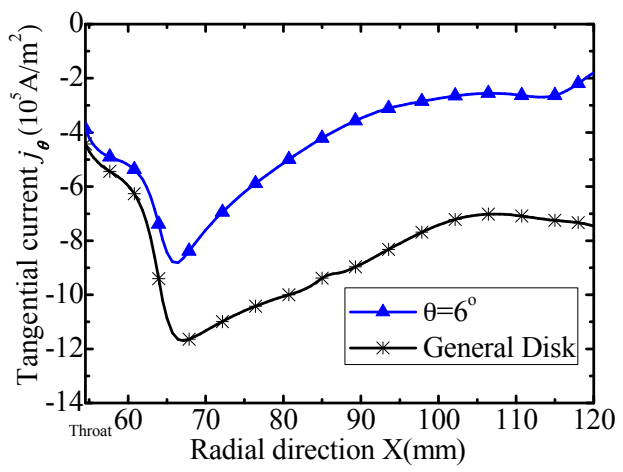

Figure 4. The comparison of tangential current distribution of the partial disk generator and general disk generator along radial direction.

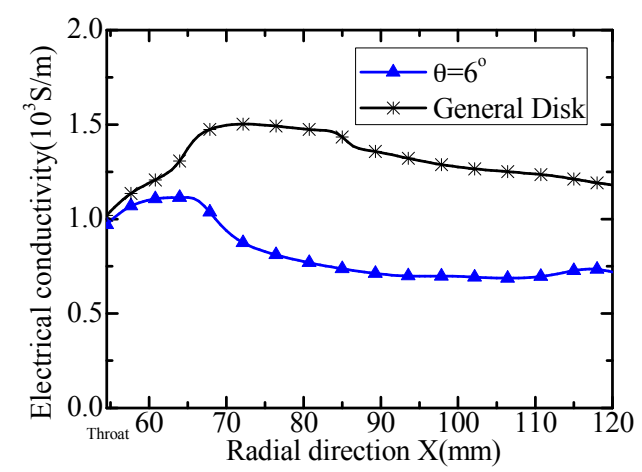

Figure 5. The comparison of electrical conductivity distribution of the partial disk generator and general disk generator along radial direction. 
In Figure 6, the radial velocity of the partial disk generator is consistently larger than that of the general disk generator at the region $65-120 \mathrm{~mm}$. Because of the strength of Lorentz force $j_{\theta} B$, in the reverse direction of radial velocity, is lower than that in the general disk channel, the tangential current is weakened in the division channel, which can be seen in Figure 4. The static pressure diminishes more sharply in the partial disk generator than in the general disk generator because of the diminishing of Lorentz force in the partial disk generator (Figure 7). In conclusion, the conjectures ahead of this section are confirmed from Figures 4-7.

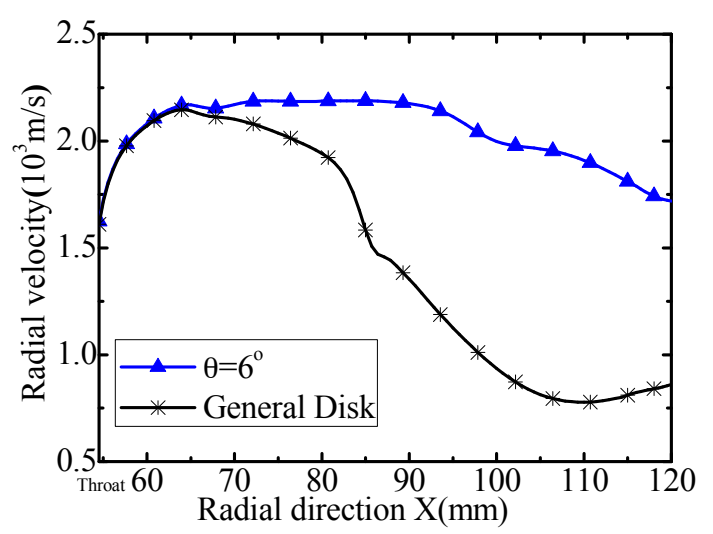

Figure 6. The comparison of radial velocity between the partial disk generator and general disk generator.

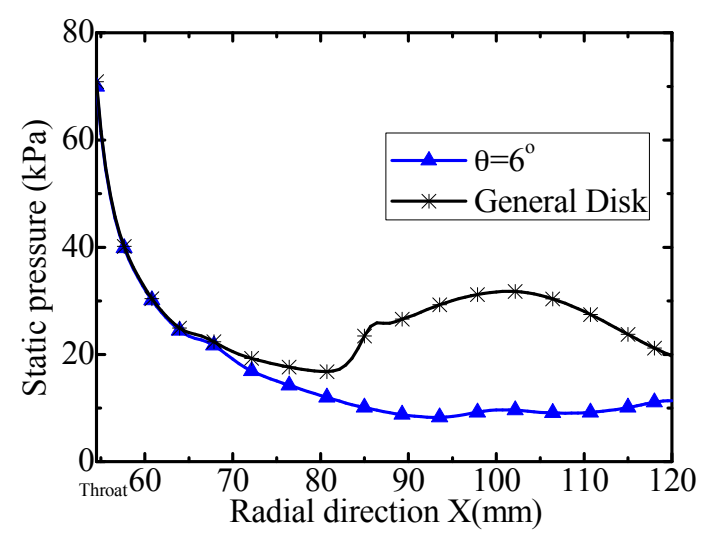

Figure 7. The comparison of static pressure between the partial disk generator and general disk generator.

Generally, low static pressure indicates high gas velocity because of the high dynamic pressure. The gas is more uniform when the gas velocity under little changing. On the contrary side, gas flow is decelerated by strong Lorentz force and its kinetic energy is converted into pressure results in the increase of static gas pressure, the non-uniform discharge structure, and local increase of eddy current likely arise in those regions. The main cause in ionization instability is local non-uniform discharge results in the increase of electron temperature, hence, the electron number density burst into high level in the local position, which further motivates the non-uniform discharge, the arise of eddy current, and the non-uniform ionization. The electron temperature distributions of the partial disk channel and general disk channel are described in Figure 8. The electron temperature ranges of the general disk channel is $9000-12,000 \mathrm{~K}$ due to the stronger Joule heating effect. That in the partial channel is only $5500-8500 \mathrm{~K}$. Therefore, the electron number density increases along the general disk channel (Figure 9a). The electron number density is larger, especially around cathode, because of the strong Joule heating effect near the electrode. This could generate the electric arc and result in the 
erosion of the electrode. On the other side, in the partial disk channel, Joule heating is weak, while the radial velocity is high, so the electron temperature is relatively low (about $5500 \mathrm{~K}$ near the cathode). Meanwhile, the electron number density decreases moderately along the channel (Figure 9b), which means the plasma is under a recombination state. The recombination plasma realized at a high total inlet temperature can provide uniform and stable plasma, and suppress the ionization instability [10]. Hence, the partial disk channel can suppress ionization instability and provide more stable plasma.

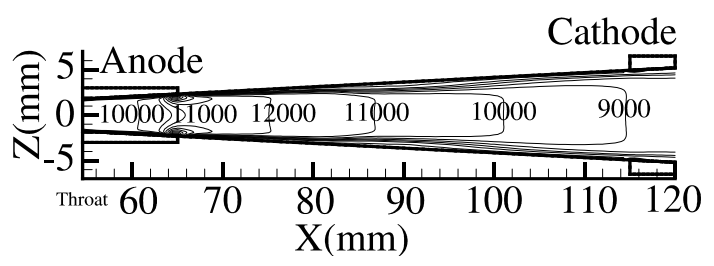

(a)

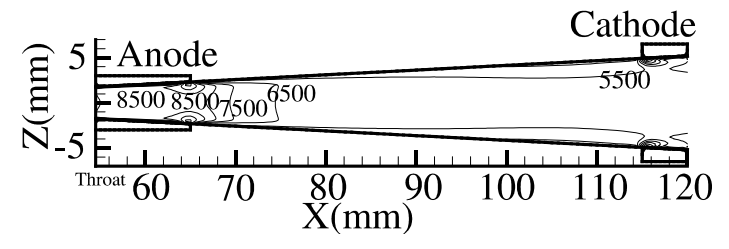

(b)

Figure 8. Distribution of electron temperature (K): (a) In the general disk channel; and, (b) In the partial disk channel.

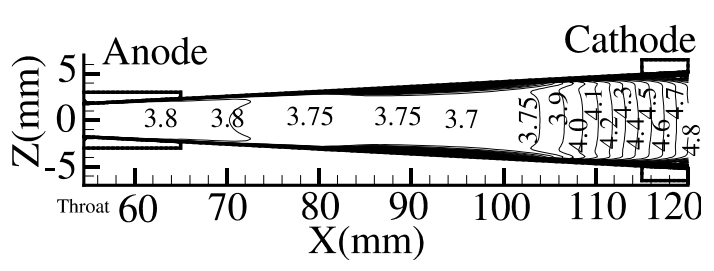

(a)

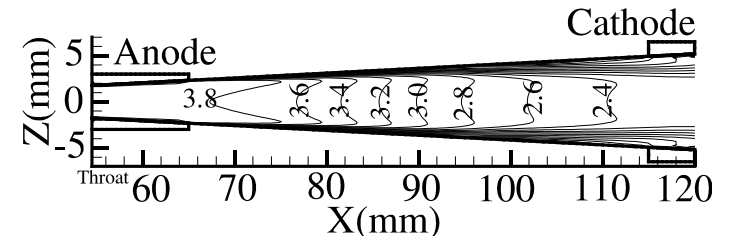

(b)

Figure 9. Distribution of electron number density $\left(10^{21} / \mathrm{m}^{3}\right)$ : (a) In the general disk channel; and, (b) In the partial disk channel.

As described at the front of this section, that more thermal energy is converted into Joule heating in general disk generator than in the partial disk generator. That means more power can be extracted in the partial disk generator. The enthalpy extraction ratio is defined as

$$
\eta_{g}=\int_{V}|\mathbf{j} \cdot \mathbf{E}| d V /\left[m_{0}\left(c_{p} T+u_{0}^{2} / 2\right)\right]
$$

where $m_{0}$ is the mass flow rate and electrical efficiency is defined as $\eta_{e}=|\mathbf{j} \cdot \mathbf{E}| /|\mathbf{u} \cdot(\mathbf{j} \times \mathbf{B})|$, where $j^{2} / \sigma=-\mathbf{u} \cdot(\mathbf{j} \times \mathbf{B})+\mathbf{j} \cdot \mathbf{E}$ of the partial and general disk generator are shown in Table 3. It declares that the partial disk channel is effective to improve the power generating performance.

Table 3. Enthalpy extraction ratio and electrical efficiency.

\begin{tabular}{ccccccc}
\hline Angle $^{\circ}$ & 4 & 5 & 6 & 10 & 15 & General \\
Enthalpy extraction ratio \% & 21.19 & 23.26 & 23.07 & 23.16 & 21.3 & 10.4 \\
Electrical efficiency \% & 78.29 & 79.97 & 77.09 & 69.13 & 60.77 & 21.8 \\
\hline
\end{tabular}

\subsection{Discussion of Optimal Angle of Partial Disk Generator}

The optimal division angle of the partial disk mainly depends on two factors: electrical efficiency and feasibility. On the one hand, the generator is expected to convert the thermal energy of a working fluid into electrical power as much as possible. On the other hand, the generator should have the brief structure as the general disk does. From the discussions in part 2, the performance of the generator with smaller division angle is better. However, the smaller division angle needs more parts to build up a whole disk, which is against the principle of practice and feasibility. Consequently, the 4 degrees is 
the minimum angle for the study of the channel model. To obtain the optimal angle degree for the generator, the difference between the angles is $1,2,4,5,6,9,10$, and 11. The performance of the partial disk generator with 5, 6, 10, and 15 degrees is simulated.

Figure 10 shows that each radial velocity is close from throat to $95 \mathrm{~mm}$ due to high speed and low Lorenz force. At the middle and tail of the channel (95-120 mm), when compared with the 4, 5, and 6 degrees channels, the radial velocity of the 10 and 15 degrees partial disk channel decreases sharply, because the Lorenz force increases with the increase of the angle of the channel. The reason is that the tangential current increases with an enlarging of the angle. It also can be seen from Figure 11 that the static pressure increases with the expansion of the channel angle. Since the smaller static pressure is beneficial to ionization stability and generation performance, the angle of 5 to 10 degrees is preferable for stabilizing ionization of the MHD channel. When the angle exceeds 10 degrees, the static pressure boosts downstream, which means that the performance of partial channel tends to close to the general disk channel.

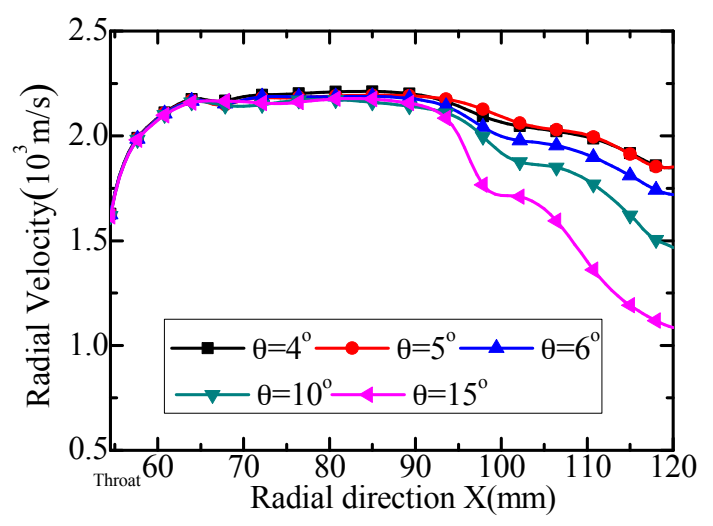

Figure 10. Distributions of radial flow velocity along the radial direction.

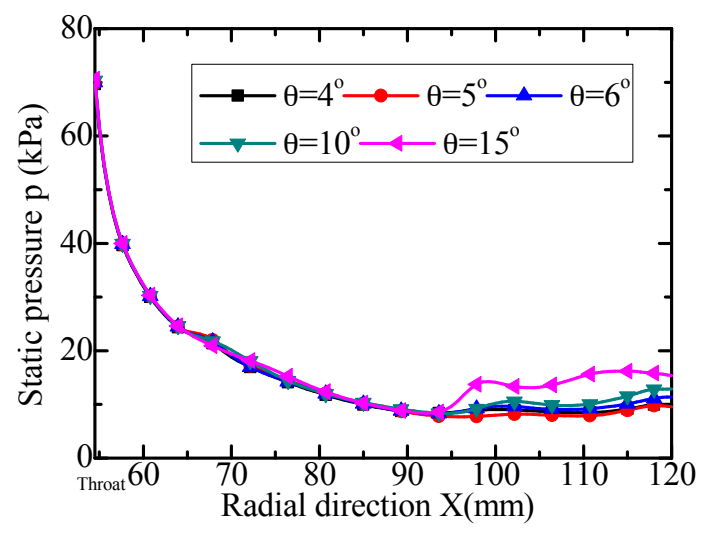

Figure 11. Distributions of static pressure along the radial direction.

An increase of the internal energy occurs in the 95-120 mm region of the partial disk channel dominated by the strong Joule heating effect. Figure 12 describes the distributions of the static temperature in those channels. The static temperature (except 5 degree) increases when the angle is enlarging. 


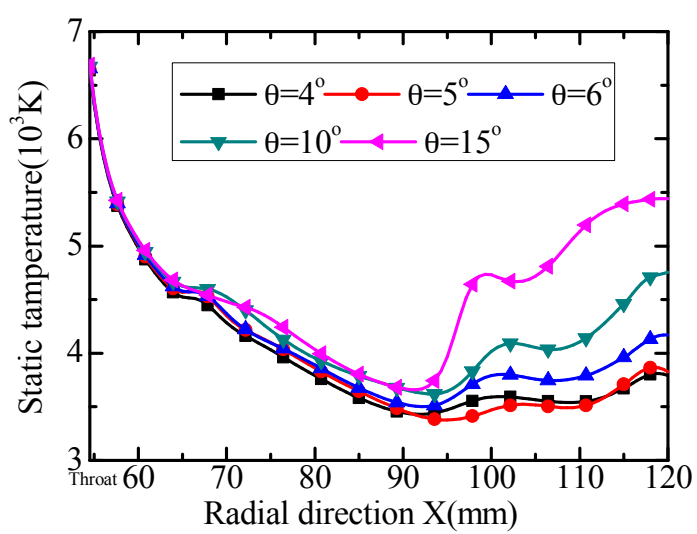

Figure 12. Distributions of static temperature along the radial direction.

With the small differences of 4, 5, 6 degrees, their distributions of the electron temperature are close. However, the channels at 10 and 15 degrees increase rapidly, and the electron temperature is up to $9000-10,000 \mathrm{~K}$ near the anode and $6000-7000 \mathrm{~K}$ near the cathode (Figure 13). As described in Section 3.1, the Joule heating is stronger in the general disk channel. The general disk channel can be considered as the extreme case, when the angle is enlarging, it is reasonable to explain that more thermal energy is converted into Joule heating in larger angle channels from Figure 13. On the other side, it is easy to form electrical eddy currents in the lager angle channel (the eddy currents are captured at $X=110 \mathrm{~mm}$ cross-section in channel of 15 degrees Figure 14), due to the high electrical conductivity (Figure 15), which leads to intensify Joule heating effect and enhance internal thermal energy. Therefore, the enthalpy extraction ratio and electrical efficiency decrease in the larger angle (over 15 degrees) channels.

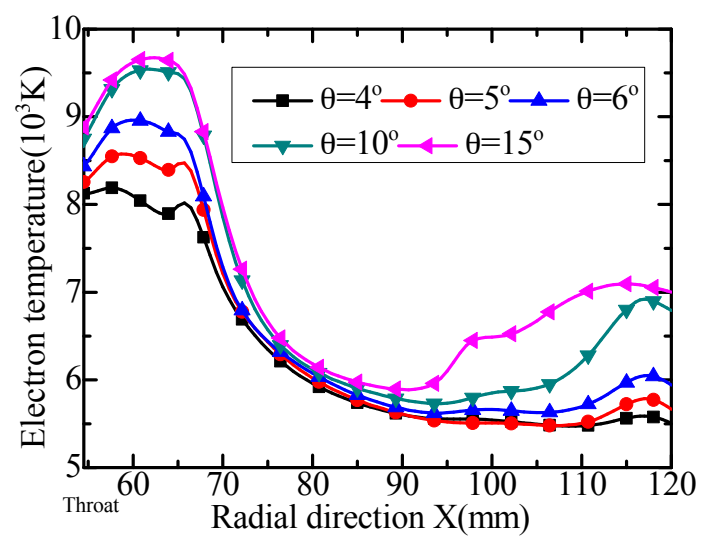

Figure 13. Distribution of electron temperature along the radial direction.

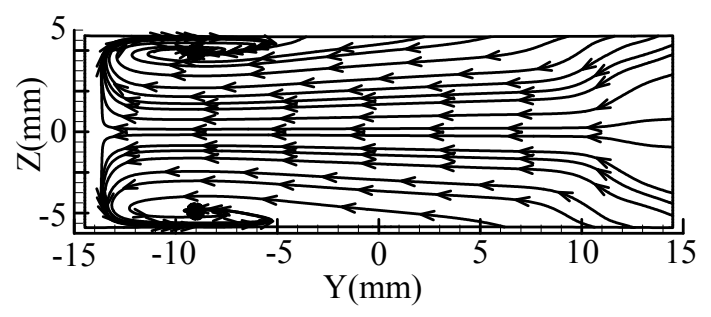

Figure 14. Eddy currents at $X=110 \mathrm{~mm}$ cross-section. 


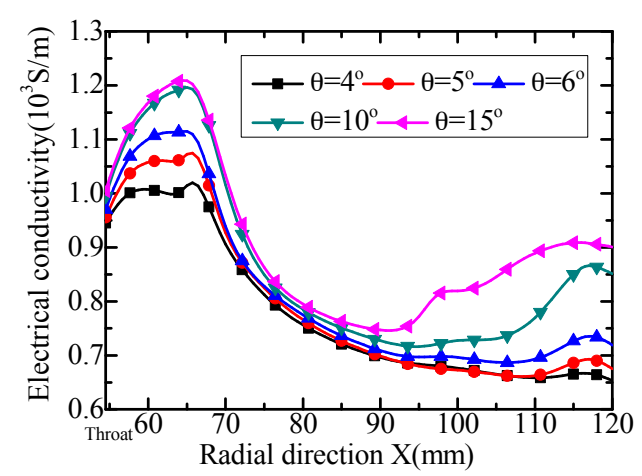

Figure 15. Distribution of electrical conductivity along the radial direction.

Figure 15 shows the electrical conductivity variation with respect to electron temperature, it demonstrates that the corresponding electrical conductivity increases when the electron temperature rises. For the electron temperature is a prominent factor for the plasma ionization, the distribution of electrical conductivity is accordance with the variation of the electron temperature.

In Hall-type generators, the Hall parameter $\beta>1$ and $j_{x}>j_{y}$ are the necessary conditions for effective energy conversion [1]. In accordance with Equation (10), the Hall parameter is in inverse proportion to the sum of average momentum transfer collision frequency (Figure 16). Figure 17 shows the Hall parameter is about 1.5 to 2.5 in the general disk channel greater than that in the partial disk channel from 55 to $110 \mathrm{~mm}$. It is less than that in the partial disk channel from 110 to $120 \mathrm{~mm}$. This is because, as shown in Figure 18a, the momentum transfer collision frequency $\left(v_{A r^{+}-e}\right)$ of ions-electrons $\left(\mathrm{Ar}^{+}-e\right)$ is dominant in the two channels.

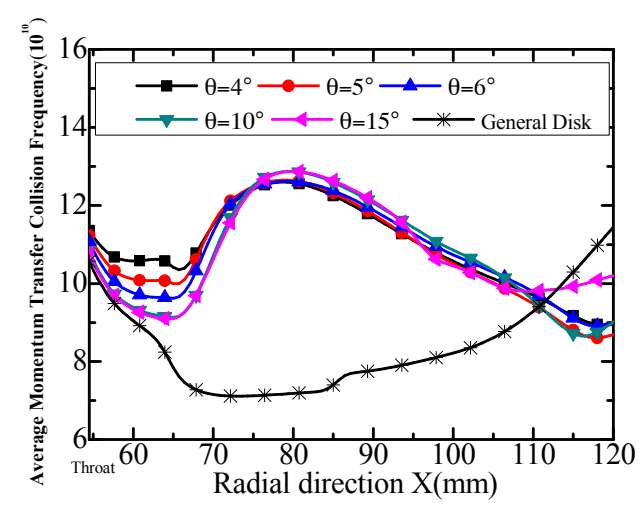

Figure 16. Distribution of sum of average momentum transfer collision frequency $\left(10^{10}\right)$ along the radial direction.

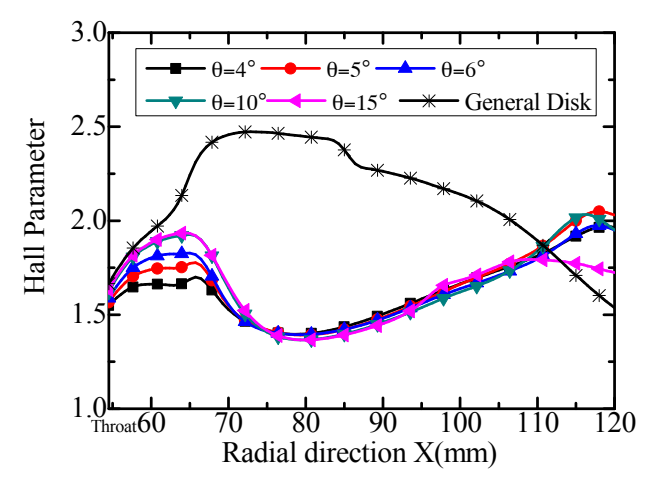

Figure 17. Distribution of Hall parameter along the radial direction. 


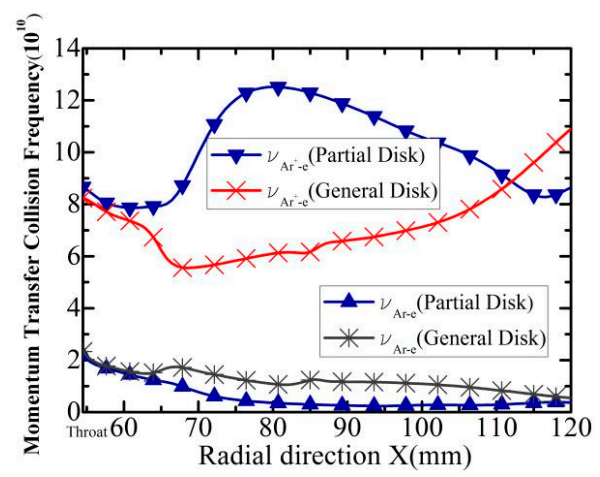

(a)

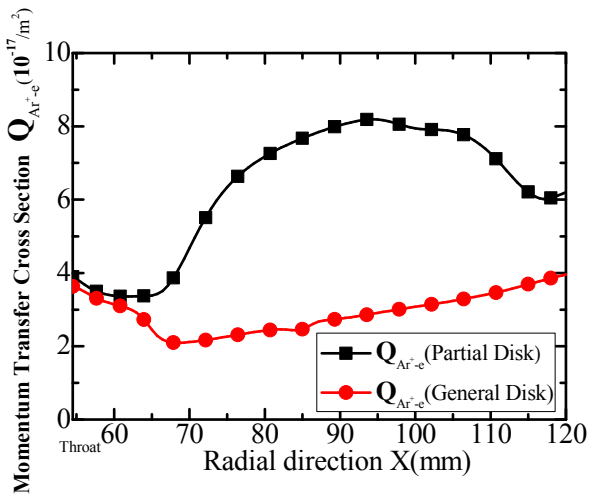

(b)

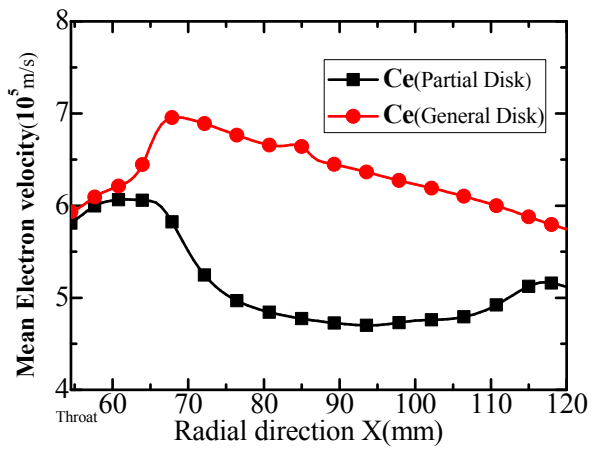

(c)

Figure 18. Distribution of terms of average momentum transfer collision frequency $\left(10^{10}\right)$ along the radial direction: (a) Distribution of $\mathrm{Ar}^{+}-e$ and $\mathrm{Ar}-e$ momentum transfer collision frequency $\left(10^{10}\right)$; (b) Distribution of momentum transfer cross section of ions-electrons $\left(10^{-17} / \mathrm{m}^{2}\right)$; and, (c) Distribution of mean electron velocity $\left(10^{5} \mathrm{~m} / \mathrm{s}\right)$.

In Figure 18a, the upper two lines represent the momentum transfer collision frequency $\left(v_{A r^{+}-e}=n_{i}^{+} Q_{A r^{+}-e} C_{e}\right)$ of ions-electrons $\left(\mathrm{Ar}^{+}-e\right)$, the lower two lines represent the momentum transfer collision frequency $\left(v_{A r-e}=n_{i} Q_{A r-e} C_{e}\right)$ of neutral particles-electrons $(\mathrm{Ar}-e)$ in two kinds of channels, respectively. From Figure 18b, the difference value of momentum transfers cross section $\left(Q_{A r^{+}-e}\right)$ of ions-electrons at the region of 55 to $110 \mathrm{~mm}$ is about $0.2-5.5$ between the partial disk channels and the general disk channel, the difference between the mean electron velocity of the two channels is 0.1-2.3 ( $C_{e}$ in Figure 18c) and the difference between the electron number density of the two channels is $0-1.5$. Hence, the value of $v_{A r^{+-} e}$ in the partial disk channels is the larger one in these areas. The electron number density $n_{e}$ increases due to the strong Joule heating effect in the general disk channel at the regions from 110 to $120 \mathrm{~mm}$, and the gap of $Q_{A r^{+}-e}$ shrinks between the two channels, so that the value of $v_{\mathrm{Ar}^{+}-e}$ increases in the general disk channel much more than that in the partial disk channels, as can be seen from Figure 18a.

The output power density $P$ of a Hall-effect MHD generator is expressed as follows [20]:

$$
P=\frac{\beta^{2}}{1+\beta^{2}} \sigma u_{r}^{2} B^{2} k_{h}\left(1-k_{h}\right), k_{h}=\frac{-E_{r}}{\beta u_{r} B}
$$

From the above equations, the term $\frac{\beta^{2}}{1+\beta^{2}}$ of the partial disk channels changes in the range of 0.69-0.8 with the Hall parameter changing in Figure 17. The electrical conductivity of each channel varies from 650 to $1200 \mathrm{~S} / \mathrm{m}$ in Figure 15. However, the radial velocity $u_{r}$ is a second-order term, the difference of $u_{r}^{2}$ ranges from $10^{2}$ to $10^{4}$, according to Figure 6 . So, the radial velocity has more influence on the performance of the generator than the electrical conductivity and the Hall parameter 
does, which is also verified from the result of Table 3. As a result, there will be a higher power output when the angle is at $5-10$ degrees.

\section{Conclusions}

In this paper, we propose a new designed structure of the disk MHD generator to investigate the stability of plasma and generator performance. Firstly, the results show the differences of main flow behaviors and plasma characteristics between the designed generator and the general disk generator. Then, the optimal degrees of performing well are found. The following conclusions are drawn from the simulations:

1. In the designed partial disk channel, the strength of Lorentz force is lower than that of the general disk channel, so the radial velocity is higher, and the static pressure is smaller. The plasma prefers to stay in a recombination process, which is beneficial to plasma uniformity and ionization stability. Moreover, the structure is conducive to obtain a high power output by diminishing energy losses of Joule heating. Consequently, higher enthalpy extraction ratio and electrical efficiency are achieved.

2. The optimum enthalpy extraction and electrical efficiency can be achieved when the angle is from 5 to 10 degrees among those partial disk channel. After the angle exceeds 15 degrees, the generator performance is clearly in a decline tendency and close to the general disk generator.

3. Different from that in the general disk channel, the Joule heating effect is weak in the partial disk channels, so the electron number density decreases moderately downstream. The electron number density is higher in upstream near the anode than that in downstream, it seems to be a source to keep the electron number density in uniform distribution downstream near the cathode, which is beneficial to electrical power output, instead of converting internal energy.

Acknowledgments: The authors appreciate the reviewers for their constructive comments and suggestions which greatly improve the manuscript. The authors also would like to thank editors for informing the progress. The current research was supported by the Fundamental Research Funds for the Central Universities, No. NP2016205.

Author Contributions: Lai Li Conceived and designed the work that led to the submission, acquired data, and interpreted the results in the manuscript. Hu-lin Huang Analyzed the feasibility, reviewed the manuscript and gave the suggestions of revising, and approved the final manuscript. Gui-ping Zhu Analyzed the feasibility, reviewed the manuscript and gave the suggestions of revising.

Conflicts of Interest: The authors declare no conflict of interest.

\section{References}

1. Rosa, R.J. Magnetohydrodynamic Energy Conversion; McGraw-Hill: New York, NY, USA, 1968.

2. Liberati, A.; Murakami, T.; Okuno, Y.; Yamasaki, H. Numerical simulation on performance of disk MHD generator in the closed-loop experimental facility. IEEE Trans. Plasma Sci. 2006, 34, 2669-2677. [CrossRef]

3. Liberati, A.; Okuno, Y. Influence of anode-region boundary-layer separation on disk MHD-generator performance. IEEE Trans. Plasma Sci. 2007, 3, 1588-1597. [CrossRef]

4. Harada, N.; Tashiro, T. Influence of Recombination Coefficient on Discharge Structure and Plasma Stability in Closed Cycle MHD Generator with He/Xe Working Gas. In Proceedings of the AIAA Plasma dynamics and Lasers Conference, Orlando, FL, USA, 23-26 June 2003. [CrossRef]

5. Harada, N.; Hishikawa, M.; Nara, N.; Sakamoto, N. Plasma Stability, Generator Performance and Stable Operation of Mixed Inert Gas Non-Equilibrium MHD Generator; MISM: Moscow, Russia, 2005; Volume 1, pp. 59-69. Available online: http://mhd.ing.unibo.it/Old_Proceedings/2005_Moscow /Moscow\%202005/ Vol1/07\%20-\%20Plasma\%20Stability,\%20Generator\%20Performance\%20and\%20Stable\%20Operation\% 20of\%20Mixed\%20Inert\%20Gas\%20Nonequilibrium.pdf (accessed on 29 January 2015).

6. Harada, N.; Le, C.K.; Tashiro, T. Closed Cycle MHD Generator Using He/Xe Working Plasma. In Proceedings of the AIAA Plasmadynamics and Lasers Conference, Maui, HI, USA, 20-23 May 2002. [CrossRef]

7. Murakami, T.; Okuno, Y. Simulation and demonstration of magnetohydrodynamic energy conversion in a high-temperature inert gas. Phys. Plasmas 2009, 16, 425-429. [CrossRef] 
8. Murakami, T.; Okuno, Y. High-density magnetohydrodynamic energy conversion in a high-temperature inert gas. Appl. Phys. Lett. 2008, 93, 041504. [CrossRef]

9. Tanaka, M.; Okuno, Y. Performance of a seed-free disk magnetohydrodynamic generator with self-excited joule heating in the nozzle. IEEE Trans. Plasma Sci. 2017, 45, 454-460. [CrossRef]

10. Tanaka, M.; Murakami, T.; Okuno, Y. Plasma characteristics and performance of magnetohydrodynamic generator with high-temperature inert gas plasma. IEEE Trans. Plasma Sci. 2014, 42, 4020-4025. [CrossRef]

11. Liberati, A.; Okuno, Y. Numerical Simulation of High Mach Number: Low Static Pressure Plasma in a Highly Efficient Disk MHD Generator. In Proceedings of the AIAA Plasma dynamics and Lasers Conference, Seattle, WA, USA, 23-26 June 2008. [CrossRef]

12. Liberati, A.; Murakami, T.; Okuno, Y.; Yamasaki, H. Numerical Simulation of Less Divergent Disk MHD Generator with High Magnetic Flux Density. In Proceedings of the AIAA Plasmadynamics and Lasers Conference, San Francisco, CA, USA, 5-8 June 2006. [CrossRef]

13. Murakami, T.; Okuno, Y. Experiment and Simulation of MHD Power Generation Using Convexly Divergent Channel. In Proceedings of the AIAA Plasma dynamics and Lasers Conference, Honolulu, HI, USA, 27-30 June 2013; pp. 861-872. [CrossRef]

14. Harada, N. Characteristics of a disk MHD generator with inlet swirl. Energy Convers. Manag. 1995, 40, 305-318. [CrossRef]

15. Liu, H.; Xu, K.; Zhu, T.; Ye, W. Multiple temperature kinetic model and its applications to micro-scale gas flows. Comput. Fluids 2012, 67, 115-122. [CrossRef]

16. Zhu, T.; Ye, W. Theoretical and numerical studies of noncontinuum gas-phase heat conduction in micro/nano devices. Numer. Heat Transfer Part B Fundam. 2010, 57, 203-226. [CrossRef]

17. Aidun, C.K.; Clausen, J.R. Lattice-Boltzmann method for complex flows. Annu. Rev. Fluid Mech. 2010, 42, 439-472. [CrossRef]

18. Mitchner, M.; Kruger, C.H. Partially Ionized Gases; Wiley: New York, NY, USA, 1973.

19. Zheng, X.; Haoyu, L.U.; Dajun, X.U.; Guobiao, C.A.I. Numerical simulation of 2D supersonic magnetohydrodynamic channel and study on hall effect. Chin. J. Aeronaut. 2011, 24, 136-144. [CrossRef]

20. Sakai, T.; Matsumoto, M.; Murakami, T.; Okuno, Y. Numerical simulation of power generation characteristics of a disk MHD generator with high-temperature inert gas plasma. Electr. Eng. Jpn. 2012, 179, 23-30. [CrossRef]

21. Biberman, L.M.; Vorob'Ev, V.S.; Yakubov, I.T. On the theory of ionization and recombination in a low-temperature plasma. Sov. J. Exp. Theor. Phys. 1969, 29, 1070-1073.

22. Tanaka, M.; Murakami, T.; Okuno, Y. Numerical simulation of performance of high-temperature inert gas plasma faraday-type magnetohydrodynamic generator. J. Propuls. Power 2015, 38, 1-8. [CrossRef]

23. Tanaka, M.; Zhuang, Y.; Komatsu, F.; Murakami, T.; Okuno, Y. Power Generation Experiments with a High Temperature Inert Gas Plasma Faraday Type MHD Generator. In Proceedings of the AIAA Plasma dynamics and Lasers Conference, San Diego, CA, USA, 24-27 June 2013; Volume 134, pp. 58-65. [CrossRef]

24. Tanaka, M.; Murakami, T.; Okuno, Y. Plasma fluid flow behavior and power generation characteristics in a high-temperature inert gas plasma faraday MHD generator. Electr. Eng. Jpn. 2016, 194, 46-53. [CrossRef]

25. Okuno, Y.; Watanabe, K.; Kawasaki, A.; Murakami, T. Experimental Studies of Seed-Free Pure-Inert-Gas Working MHD Power Generation. In Proceedings of the AIAA Plasma Dynamics and Lasers Conference, Honolulu, HI, USA, 27-30 June 2011. [CrossRef]

26. Litchford, R.J.; Harada, N. Multi-MW Closed Cycle MHD Nuclear Space Power Via Nonequilibrium He/Xe Working Plasma. In Proceedings of the Nuclear and Emerging Technologies for Space, Albuquerque, NM, USA, 7-10 February 2011.

27. Tanaka, M.; Okuno, Y. High-Temperature Inert-Gas-Plasma Faraday-Type Magnetohydrodynamic Generator with Various Working Gases. J. Propuls. Power 2016, 32, 1-6. [CrossRef]

28. Ichinokiyama, D.; Takayasu, F. Numerical Analysis of Non-equilibrium Disk MHD Generator with Swirl Vanes. In Proceedings of the International Energy Conversion Engineering Conference, Salt Lake City, UT, USA, 25-27 July 2016. [CrossRef]

(C) 2018 by the authors. Licensee MDPI, Basel, Switzerland. This article is an open access article distributed under the terms and conditions of the Creative Commons Attribution (CC BY) license (http:/ / creativecommons.org/licenses/by/4.0/). 